ARTICLE

Received 31 Oct 2014 | Accepted 11 Jun 2015 | Published 17 Jul 2015

DOI: $10.1038 /$ ncomms 8800

OPEN

\title{
Noble metal-comparable SERS enhancement from semiconducting metal oxides by making oxygen vacancies
}

Shan Cong ${ }^{1, \star}$, Yinyin Yuan ${ }^{1,2, \star}$, Zhigang Chen ${ }^{1}$, Junyu Hou ${ }^{3}$, Mei Yang ${ }^{1}$, Yanli Su${ }^{3}$, Yongyi Zhang ${ }^{1}$, Liang $\mathrm{Li}^{2}$, Qingwen $\mathrm{Li}^{1}$, Fengxia Geng ${ }^{3} \&$ Zhigang Zhao ${ }^{1}$

Surface-enhanced Raman spectroscopy (SERS) represents a very powerful tool for the identification of molecular species, but unfortunately it has been essentially restricted to noble metal supports ( $\mathrm{Au}, \mathrm{Ag}$ and $\mathrm{Cu}$ ). While the application of semiconductor materials as SERS substrate would enormously widen the range of uses for this technique, the detection sensitivity has been much inferior and the achievable SERS enhancement was rather limited, thereby greatly limiting the practical applications. Here we report the employment of non-stoichiometric tungsten oxide nanostructure, sea urchin-like $\mathrm{W}_{18} \mathrm{O}_{49}$ nanowire, as the substrate material, to magnify the substrate-analyte molecule interaction, leading to significant magnifications in Raman spectroscopic signature. The enrichment of surface oxygen vacancy could bring additional enhancements. The detection limit concentration was as low as $10^{-7} \mathrm{M}$ and the maximum enhancement factor was $3.4 \times 10^{5}$, in the rank of the highest sensitivity, to our best knowledge, among semiconducting materials, even comparable to noble metals without 'hot spots'.

\footnotetext{
${ }^{1}$ Key Lab of Nanodevices and Applications, Suzhou Institute of Nano-Tech and Nano-Bionics, Chinese Academy of Sciences (CAS), Suzhou 215123, China.

${ }^{2}$ Key Laboratory for Ultrafine Materials of Ministry of Education, School of Materials Science and Engineering, East China University of Science and Technology, Shanghai 200237, China. ${ }^{3}$ College of Chemistry, Chemical Engineering and Materials Science, Soochow University, Suzhou 215123, China.

* These authors contributed equally to this work. Correspondence and requests for materials should be addressed to Z.Z. (email: zgzhao2011@sinano.ac.cn).
} 
T he surface-enhanced Raman scattering (SERS) effect is a surface-sensitive technique characterized by an increase in the Raman intensity by orders of magnitude for molecules adsorbed on particular surfaces such as rough metals with respect to that expected from the same number of non-adsorbed molecules in solution or the gas phase ${ }^{1,2}$. The enhanced sensitivity makes the technique serve as one of the most powerful analytical tools for the unequivocal identification of chemical and biological analytes and additionally in numerous other fields, such as electrochemistry, surface science, catalysis, chemical and biomolecular sensing and so on ${ }^{1,3,4}$. While the exact mechanism for the enhancement effect is still a matter of debate, two widely accepted theories have been adopted in most cases to explain the phenomenon ${ }^{5}$. The primary one is electromagnetic mechanism predicting that the electric field is magnified when excitation takes place within localized surface plasmon resonances of substrate materials, leading to enhancement factors (EFs) up to the order of $10^{6}$. The induced enhancement of local field by plasmonic coupling is called electromagnetic 'hot spots'. The other is a chemical mechanism proposing the formation of charge-transfer complexes between the chemisorbed species and the substrate materials and obtaining enhancement when the excitation frequency is in resonance with the chargetransfer transition with EFs around 10-100. SERS experiments have been essentially dominated by adsorbates on rough metallic surfaces, especially noble and alkali metals such as $\mathrm{Au}, \mathrm{Ag}$ and $\mathrm{Cu}$, because their plasmon resonance frequencies locate within excitation wavelength ranges commonly used in Raman spectroscopy. While precise tuneable position of adjacent metallic nanoparticles is a key factor to bring about strong plasmonic coupling, fabricating perfect plasmonic nanostructures with high density 'hot spots' to achieve high enhancements usually requires delicate procedures and high cost. In addition, noble metals typically show poor stability and biocompatibility, and therefore the search of other alternative materials for working as SERS substrate becomes an urgent task. A few semiconducting materials have been proven to show Raman enhancement, for example, InAs/GaAs quantum dots ${ }^{6}, \mathrm{CuTe}$ nanocrystals ${ }^{7}, \mathrm{Cu}_{2} \mathrm{O}$ nanospheres ${ }^{8}$ and $\mathrm{TiO}_{2}$ nanostructures ${ }^{9,10}$, in which charge transfer at the semiconductor-analyte interface plays a major role in Raman scattering enhancement. The $\mathrm{TiO}_{2}$ SERS substrate offers not only a biocompatibility, but also a greater chemical and mechanical stability against variation in $\mathrm{pH}$ or temperature of the environment ${ }^{9}$. The effective utilization of semiconductor SERSactive substrates would greatly expand the applications of SERS in many fields, such as direct monitoring of interfacial chemical reactions on individual nanoparticles ${ }^{11}$. However, one significant problem of metal-free SERS-active substrates is that the EFs were typically quite low, usually in the range of $10-10^{2}$, far from sufficient for applications in biological and biomedical analysis and diagnosis. It is consequently one formidable challenge but greatly desirable to find efficient semiconductor SERS substrate materials and obtain EFs comparable to noble metals.

The surface states of substrate material and its interaction with analyte molecule is a key to the SERS effect. Among the numerous methods in tuning the surface states of semiconducting oxide materials, adjusting oxygen deficiency represents one main strategy and may work as an efficient and simple way to achieve the goal of high sensitivity comparable to that of noble metals. Tungsten oxide materials, a traditional semiconductor, have attracted considerable attention because they possess distinctive physical and chemical properties, which endow them to be effective candidates in a wide range of applications, covering photocatalysts, gas sensors and electrochromic devices ${ }^{12-14}$. Importantly, rich phases of substoichiometric composition $\left(\mathrm{WO}_{3-x}\right)$ can be obtained by the reduction and formation of various types of defect structure, such as $\mathrm{WO}_{2.72}\left(\mathrm{~W}_{18} \mathrm{O}_{49}\right)$, $\mathrm{WO}_{2.8} \quad\left(\mathrm{~W}_{5} \mathrm{O}_{14}\right), \mathrm{WO}_{2.92} \quad\left(\mathrm{~W}_{25} \mathrm{O}_{73}\right)$ and so on ${ }^{15-17}$, which makes the system work as an ideal platform for investigating the effect of vacancies or defects on SERS phenomenon. $\mathrm{WO}_{2.72}$ $\left(\mathrm{W}_{18} \mathrm{O}_{49}\right)$ with the largest vacancy has been reported as the only oxide that can be isolated in a pure form, which contains tungsten ions of mixed valency ${ }^{16,18}$.

Herein, employing vacancy-containing $\mathrm{W}_{18} \mathrm{O}_{49}$ as the substrate material, we achieve greatly enhanced SERS effect for the first time on function-rich tungsten oxide material, and the enhancement is further improved by creating surface deficiencies, which gives SERS EF as high as $3.4 \times 10^{5}$ and $100-10,000$ times higher than the previously reported values for most other semiconductor SERS-active substrates, and even comparable to noble metals without 'hot spots'.

\section{Results}

Sample characterizations. $\mathrm{W}_{18} \mathrm{O}_{49}$ was synthesized using a previously reported approach, $\mathrm{WCl}_{6}$ dissolved in ethanol followed by hydrothermal reaction at $180^{\circ} \mathrm{C}$ for $24 \mathrm{~h}$ (ref. 13). Phase and purity of the sample was verified by X-ray diffraction characterizations as shown in Fig. 1a. The hydrothermally synthesized sample was crystallized in a monoclinic phase of $\mathrm{W}_{18} \mathrm{O}_{49}(P 2 / m$, JCPDS no. 84-1516) with lattice constants refined to be $a=18.31(2), b=3.839(8), c=14.00(1) \AA$ and $\beta=115.19(9)^{\circ}$. The relatively high intensities and narrowing exhibited by (010) and (020) peaks suggest that the crystals preferably grew along the $b$ axis as a result of retarded growth along the close-packed (010) planes. For comparison study, tungsten trioxide, $\mathrm{WO}_{3}$, was acquired by annealing the $\mathrm{W}_{18} \mathrm{O}_{49}$ sample in air. The transformed $\mathrm{WO}_{3}$ was also of high purity with all reflections perfectly fitted in stoichiometric monoclinic (no. 14, $\left.P 2_{1} / n\right)$ tungsten trioxide with cell dimensions $a=7.305(5)$, $b=7.523(4), c=7.689(4) \AA$ and $\beta=90.90(9)^{\circ}$. To obtain more information about the intrinsic nature of the samples, ultravioletvisible absorption spectra of the two samples were collected (Fig. 1b). Distinct from the absorption to $500 \mathrm{~nm}$ normally observed in tungsten oxide materials, $\mathrm{W}_{18} \mathrm{O}_{49}$ sample exhibited an obvious blue shift of the absorption edge. In addition, an absorption tail beyond the absorption edge in the visible and near-infrared region was observed, up to the measurement limit, $800 \mathrm{~nm}$, which can be considered to be closely related with the free electrons and/or oxygen deficiency-induced small polarons ${ }^{15}$, providing clear evidence that the as-synthesized $\mathrm{W}_{18} \mathrm{O}_{49}$ sample contains a large number of oxygen vacancies. Morphology of the samples were characterized by scanning electron microscope observations, which suggested that the as-grown $\mathrm{W}_{18} \mathrm{O}_{49}$ sample comprised of thin nanowires with average length estimated to be about several micrometres while width in the range of $10-20 \mathrm{~nm}$ (Fig. 1c). The nanowires were entangled featuring a prickly, spherical and sea urchin-like morphology. High-resolution transmission electron microscopy on one single nanowire discerned clear lattice fringes belonging to (010) planes of monoclinic $\mathrm{W}_{18} \mathrm{O}_{49}$ with an interplanar spacing of $\sim 0.38 \mathrm{~nm}$ (inset in Fig. 1c), which undoubtedly indicates that the nanowires preferably grow along the [010] direction, in good agreement with $\mathrm{X}$-ray diffraction observations. After annealing in air and oxidizing into $\mathrm{WO}_{3}$, the morphology was featureless, showing irregular particles of several micrometres (Fig. 1d).

The defect structure of $\mathrm{W}_{18} \mathrm{O}_{49}$ is illustrated in Fig. 2, in comparison with the perfect structure of $\mathrm{WO}_{3}$, projected along the [010] direction. The crystal structure of $\mathrm{WO}_{3}$ consists of slabs of corner-sharing $\mathrm{WO}_{6}$ octahedra $\left(\mathrm{ReO}_{3}\right.$-type), which have an infinite extension in two dimensions (ac plane) and a finite, characteristic width in the [010] direction. The slabs are mutually 

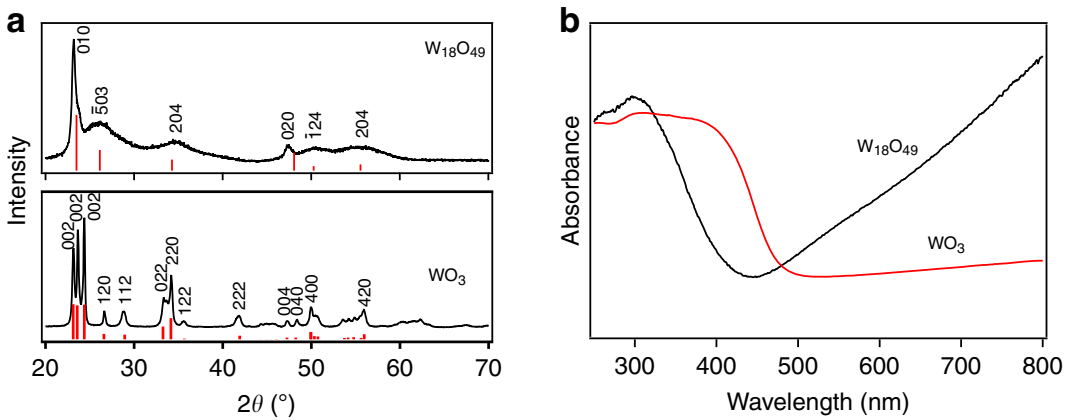

c

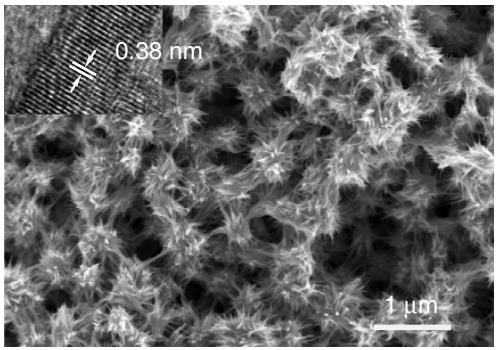

d

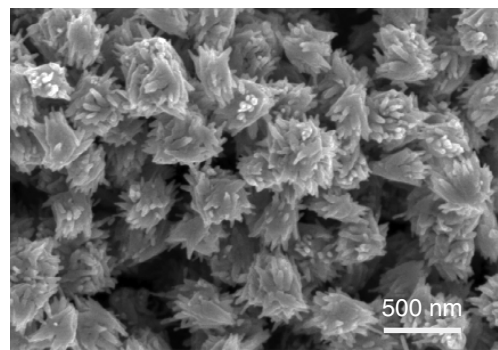

Figure 1 | Characterization of $\mathbf{W}_{\mathbf{1 8}} \mathbf{O}_{\mathbf{4 9}}$ and annealed $\mathbf{W O}_{\mathbf{3}}$ samples. (a) X-ray diffraction patterns of as-obtained $\mathrm{W}_{18} \mathrm{O}_{49}$ sample and annealed $\mathrm{WO}_{3}$ with all reflections perfectly indexed; (b) ultraviolet-visible profile comparison between $\mathrm{W}_{18} \mathrm{O}_{49}$ sample and annealed $\mathrm{WO}_{3}$, showing a blue shift and an obvious absorption tail beyond the edge that may arise from the presence of oxygen vacancies; (c,d) scanning electron microscopy images for $\mathrm{W}_{18} \mathrm{O}_{49}$ and $\mathrm{WO}_{3}$ samples, respectively. Inset in (c): high-resolution transmission electron microscopy image on one single nanowire illustrating clear lattice fringe of $0.38 \mathrm{~nm}$, which suggested that the nanowire growth was along the [010] direction.
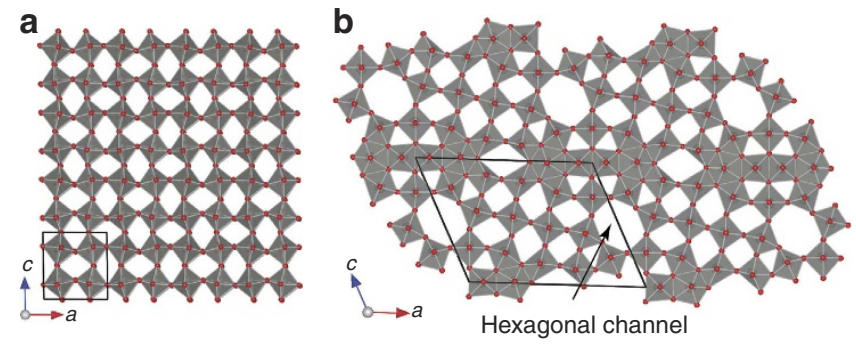

Figure 2 | Structure illustration for $\mathrm{WO}_{3}$ and $\mathrm{W}_{18} \mathrm{O}_{49}$ in [010] projection. (a) The crystalline $\mathrm{WO}_{3}$ structure consists of stacking of infinite cornersharing $\mathrm{WO}_{6}$ octahedra layers, while (b) the defects in $\mathrm{W}_{18} \mathrm{O}_{49}$ structure lead to the formation of hexagonal channels.

linked, forming channels between the octahedra. In contrast, the reduction of $\mathrm{WO}_{3}$ to $\mathrm{W}_{18} \mathrm{O}_{49}$ leads to structural changes of the $\mathrm{ReO}_{3}$-type structure and the formation of tungsten pentagonal columns causes the arising of additional hexagonal channels ${ }^{19}$. The presence of oxygen defects may enrich the surface states of semiconductor and enhance the interaction affinity between the adsorbent and adsorbate, providing promises for enhancements in Raman signals.

Raman enhancement for $\mathrm{W}_{18} \mathrm{O}_{49}$ sample. The Raman enhancement behaviour of the materials was examined using laser dye Rhodamine 6G (R6G) as a Raman probe, the molecular structure of which is provided in inset of Fig. 3a. R6G is a strongly fluorescent xanthene derivative that shows a molecular resonance effect when excited into its visible absorption band. Figure 3a shows the Raman spectra of R6G $\left(10^{-6} \mathrm{M}\right)$ with the excitation wavelength being $532.8 \mathrm{~nm}$ on substrates deposited with $\mathrm{W}_{18} \mathrm{O}_{49}$ nanowires, annealed $\mathrm{WO}_{3}$, along with bare $\mathrm{SiO}_{2} / \mathrm{Si}$. While no clear signals related with R6G molecule were discerned on bare
$\mathrm{SiO}_{2} / \mathrm{Si}$ substrate and $\mathrm{WO}_{3}$ except the fluorescence background, a substantial Raman enhancement was observed on $\mathrm{W}_{18} \mathrm{O}_{49}$, and four characteristic bands of R6G centred at $612,773,1,360$ and $1,650 \mathrm{~cm}^{-1}$, named as $\mathrm{P} 1, \mathrm{P} 2, \mathrm{P} 3$ and $\mathrm{P} 4$, were clearly detected, which suggests that $\mathrm{W}_{18} \mathrm{O}_{49}$ could work as SERS-active substrate. Raman characterizations on bare $\mathrm{W}_{18} \mathrm{O}_{49}$ in the absence of probe molecules were also performed, in which only the signals related to $\mathrm{O}-\mathrm{W}-\mathrm{O}$ bending and $\mathrm{W}-\mathrm{O}$ stretching modes were found (Supplementary Fig. 1), further confirming that the P1, P2, P3 and $\mathrm{P} 4$ bands originated from R6G molecules. P1 and P2 can be assigned to in-plane and out-of-plane bending motions of carbon and hydrogen atoms of the xanthenes skeleton, respectively; P3 and $\mathrm{P} 4$ correspond to aromatic $\mathrm{C}-\mathrm{C}$ stretching vibration modes ${ }^{20}$. Raman spectra at four different R6G concentrations (the range was selected according to adsorption isotherms in Supplementary Fig. 2), decreasing from $10^{-4}, 10^{-5}$ and $10^{-6}$ to $10^{-7} \mathrm{M}$, were collected, showing obvious intensity enhancement until an extreme dilute solution was used, $10^{-7} \mathrm{M}$, in which just detectable signal was obtained (Fig. 3b). Therefore, the detection limit for $\mathrm{W}_{18} \mathrm{O}_{49}$ material can be determined to be $10^{-7} \mathrm{M}$. Such a low detection limit enables a high sensitivity towards trace amounts of analyte molecules, and makes it possible for a systematic investigation of different vibration modes in the SERS profiles.

Two signature bands, P1 and P3, were selected for calculating EFs based on the magnification of Raman intensity compared with that on bare substrate (details in Supplementary Methods $)^{21-23}$, the variation of which was depicted in Fig. $3 \mathrm{c}$ as a function of R6G analyte concentrations $\left(C_{\mathrm{R} 6 \mathrm{G}}\right)$. It is clear that the greater enhancement occurs for P1 at each concentration, which was found to be surprisingly high as $1.9 \times 10^{5}$ with the R6G concentration of $10^{-6} \mathrm{M}$, about $100-10,000$ times higher than that previously reported for most other semiconductor SERS-active substrates (Supplementary Table 1). Furthermore, the intensity variation was not uniform and instead strongly dependent on the band identity. The selective enhancement of the 

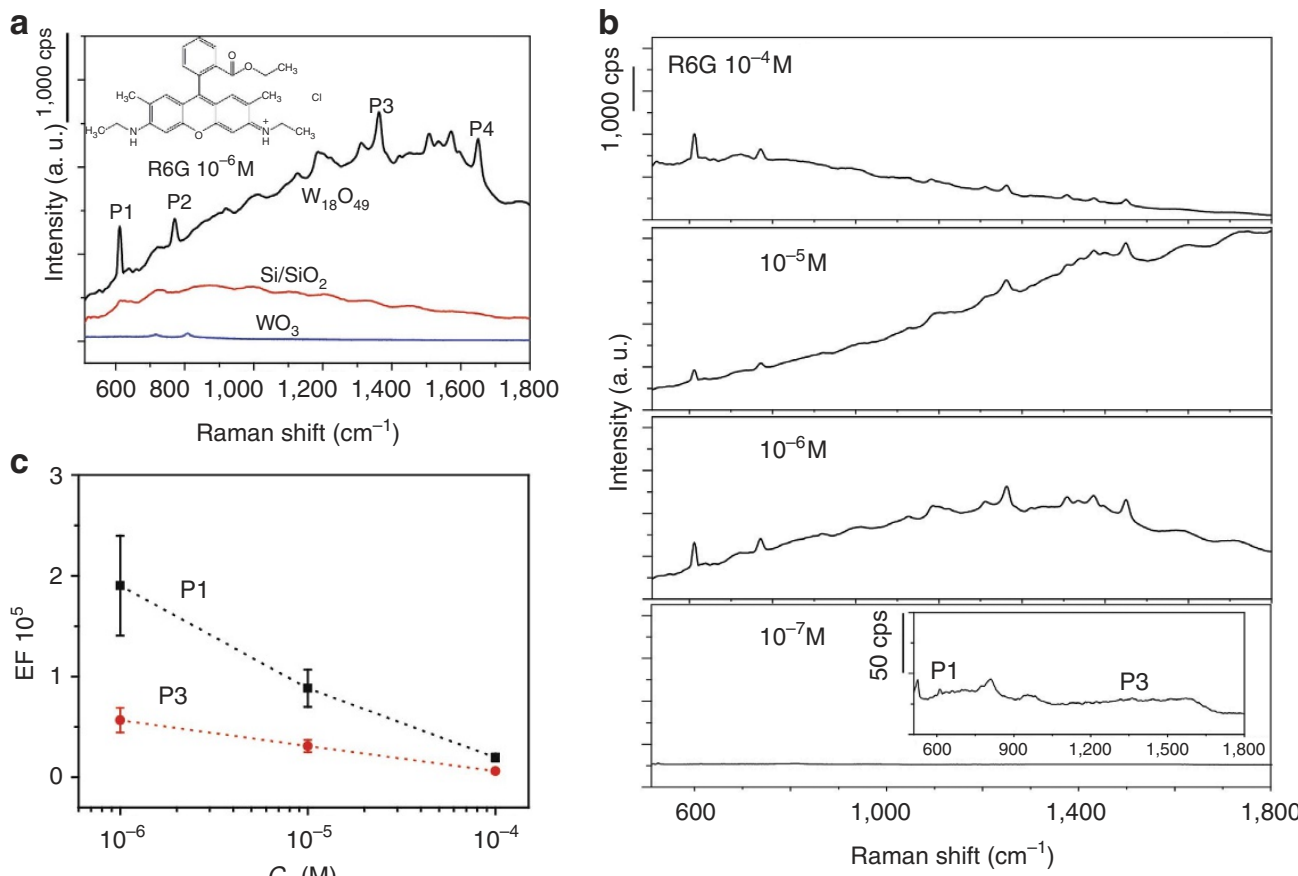

Figure 3 I SERS properties. (a) Raman profile of R6G $\left(10^{-6} \mathrm{M}\right)$ on substrates deposited with $\mathrm{W}_{18} \mathrm{O}_{49}$ sample compared with that for $\mathrm{WO}_{3}$ and bare $\mathrm{SiO}_{2} /$ Si substrate. Inset: molecule structure of R6G. (b) Raman spectra collected for $\mathrm{W}_{18} \mathrm{O}_{49}$ at four different concentrations, $10^{-4}, 10^{-5}, 10^{-6}$ and $10^{-7} \mathrm{M}_{\text {, }}$ suggesting the detection limit was as low as $10^{-7} \mathrm{M}$ (Inset: with narrowed $y$ scale for $10^{-7} \mathrm{M}$ ). (c) The statistical evolution of EF as a function of R6G concentration plotted in logarithmic scale, with the analysis carried out over 30 different regions per sample. The Raman enhancement typically increased with decreasing concentrations and selective enhancement occurred with different bands.

different vibration modes indicates that the SERS on $\mathrm{W}_{18} \mathrm{O}_{49}$ sample largely depends on the distinct binding and geometry of R6G molecules on the $\mathrm{W}_{18} \mathrm{O}_{49}$ surface, which is a character of the chemical enhancement mechanism. Specifically, the change of P1 was more obvious than that for P3. The relative lower intensity exhibited by P3, especially at low concentrations, indicates that the long axis plane of $\mathrm{R} 6 \mathrm{G}$ molecule was not parallel to the sample surface or the aromatic rings were separated by chemical groups including $\mathrm{C}-\mathrm{H}$ bond ${ }^{24}$. With dilution of the $\mathrm{R} 6 \mathrm{G}$ solutions, the signal intensity of P3 gradually increases, suggesting the gradual decrease of tilt angles of R6G molecules on the substrate. The emergence of greatly enhanced SERS sensitivity for $\mathrm{W}_{18} \mathrm{O}_{49}$ compared with $\mathrm{WO}_{3}$ can probably be attributed to the presence of oxygen vacancies in $\mathrm{W}_{18} \mathrm{O}_{49}$ structure (vide ante, Fig. 2).

Surface vacancy related Raman enhancement. To confirm the role of oxygen vacancy played in SERS, additional surface vacancy was deliberately created by annealing the $\mathrm{W}_{18} \mathrm{O}_{49}$ sample in $\mathrm{Ar} / \mathrm{H}_{2}$ atmosphere ${ }^{25}$. After the treatment at $300^{\circ} \mathrm{C}$ for $1 \mathrm{~h}$, the crystalline phase of $\mathrm{W}_{18} \mathrm{O}_{49}$ and the sea urchin-like morphology were still well kept (Fig. 4a). X-ray photoelectron spectroscopy was used to check the surface states, and Fig. 4b displays the spectra of W4f core levels for pristine $\mathrm{W}_{18} \mathrm{O}_{49}$ and the $\mathrm{Ar} / \mathrm{H}_{2}$-treated samples. The W4f core-level spectra could be deconvoluted into three doublets $\left(\mathrm{W}^{6+}, \mathrm{W}^{5+}\right.$ and $\left.\mathrm{W}^{4+}\right)$ for all samples. On the fitting analysis, it was found that the atomic percentage of $\mathrm{W}^{6+}, \mathrm{W}^{5+}$ and $\mathrm{W}^{4+}$ for pristine $\mathrm{W}_{18} \mathrm{O}_{49}$ was $54.1,30.4$ and $15.5 \%$, roughly agreeing the documented values ${ }^{26}$. By contrast, the percentage for $\mathrm{W}^{5+}$ increased to 35.4 and $47.5 \%$, $\mathrm{W}^{4+}$ to 21.4 and $13.9 \%$ for $\mathrm{Ar}$ and $\mathrm{H}_{2}$ thermal-treated samples, respectively. The increased percentage for reduced tungsten, $\mathrm{W}^{4+}$ and $\mathrm{W}^{5+}$, is likely to be related with the creation of
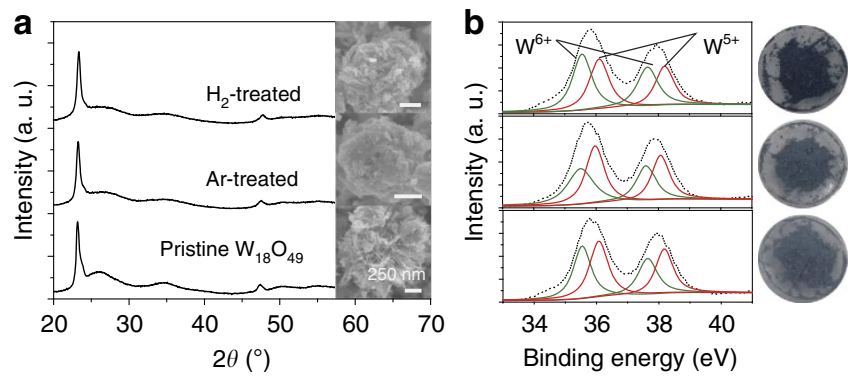

Figure 4 | Characterization of $\mathrm{W}_{18} \mathrm{O}_{49}$ samples with modulated surface oxygen vacancies. (a) X-ray diffraction patterns of $\mathrm{Ar}$ - and $\mathrm{H}_{2}$-treated samples along with that for as-prepared $\mathrm{W}_{18} \mathrm{O}_{49}$ sample for comparison purpose, indicating that the crystalline phase remained during the annealing. Inset: the corresponding SEM images showing the morphology was also unchanged. (b) XPS spectra of W4f core levels for $\mathrm{W}_{18} \mathrm{O}_{49}$ samples after treatment in $\mathrm{Ar}$ and $\mathrm{H}_{2}$ atmosphere at $300^{\circ} \mathrm{C}$ and pristine $\mathrm{W}_{18} \mathrm{O}_{49}$ sea urchin-like aggregates. Right: corresponding optical images of the three samples, showing colour change from pale blue for pristine $\mathrm{W}_{18} \mathrm{O}_{49}$ to cyan and deep blue for $\mathrm{Ar}$ - and $\mathrm{H}_{2}$-treated samples.

positively charged oxygen vacancies with accompanying charge-compensating electrons ${ }^{27}$. With increasing oxygen vacancy, the intensity of the absorption tail in the visible and near-infrared region increased (Supplementary Fig. 3), consistent with the recorded results ${ }^{28}$. The sample colour changed from pale blue for pristine $\mathrm{W}_{18} \mathrm{O}_{49}$ to cyan and deep blue for Ar- and $\mathrm{H}_{2}$-treated samples, respectively, confirming the increment of oxygen deficiencies present in the examined samples.

The Raman spectra of R6G with concentration of $1 \times 10^{-6} \mathrm{M}$ for the two treated samples are given in Fig. $5 \mathrm{a}$ along with the 
spectrum of pristine $\mathrm{W}_{18} \mathrm{O}_{49}$ sample for comparison. Both treated samples clearly manifested the four characteristic peaks of R6G molecule. Neither a new peak nor an obvious shift of the characteristic peak was detected, indicating that no formation or vanish of chemical bonds between R6G and the substrates were induced on oxygen vacancy alternation. Notably, the $\mathrm{Ar}$ - and $\mathrm{H}_{2}$-treated samples were found to display further Raman intensity increase on some degree compared with pristine $\mathrm{W}_{18} \mathrm{O}_{49}$ sample, corroborating the fact that oxygen vacancy may help increase Raman detect sensitivity. The Raman intensity of the four feature bands for all the three samples were summarized in Fig. 5b, and it is obvious that the Raman enhancement was in the order of $\mathrm{H}_{2}$-treated $\mathrm{W}_{18} \mathrm{O}_{49}>$ Ar-treated $\mathrm{W}_{18} \mathrm{O}_{49}>$ pristine $\mathrm{W}_{18} \mathrm{O}_{49}$. The $\mathrm{P} 1$ band of $\mathrm{H}_{2}$-treated $\mathrm{W}_{18} \mathrm{O}_{49}$ displayed the greatest enhancement, and the corresponding $\mathrm{EF}$ was evaluated to be $3.4 \times 10^{5}$. Such high $\mathrm{EF}$ has been rarely observed in semiconducting materials, even comparable to that for noble metals without hot spots ${ }^{20}$, which may come from the combined function of intrinsic and deliberately created oxygen deficiencies.

It seems the presence of oxygen vacancies in semiconducting oxide materials, either intrinsic ones or deliberately created on the surface, could bring significant Raman enhancement for R6G molecule. To examine the interaction between tungsten oxide materials with adsorbate molecule, ultraviolet-visible spectrum of R6G molecules deposited on $\mathrm{W}_{18} \mathrm{O}_{49}$ was collected in comparison
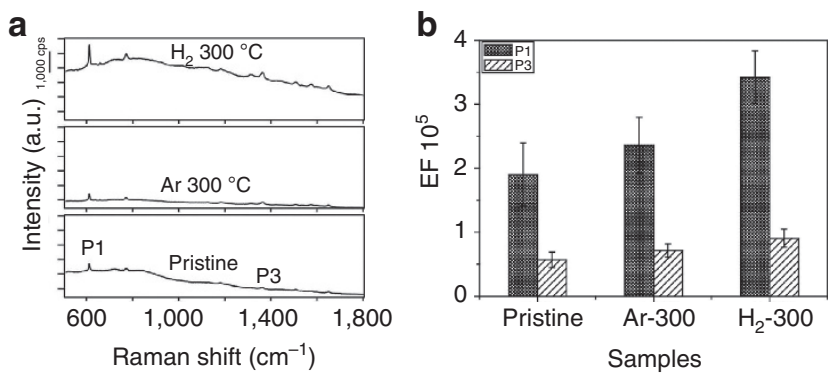

Figure 5 | Improved SERS properties of $\mathrm{W}_{18} \mathrm{O}_{49}$ samples after $\mathrm{Ar} / \mathrm{H}_{\mathbf{2}}$ annealing treatment. (a) Raman signals of $\mathrm{R} 6 \mathrm{G}$ molecule on pristine $\mathrm{W}_{18} \mathrm{O}_{49}$ and the samples after annealing treatment (in $\mathrm{Ar} / \mathrm{H}_{2}$ at $300^{\circ} \mathrm{C}$ for 1h). The tested concentration of R6G was $1 \times 10^{-6} \mathrm{M}$. (b) A comparison of Raman EF for the two respective vibration modes $\mathrm{P} 1$ and $\mathrm{P} 3$. Data reported in this histogram resulted from Raman spectra acquired over 30 different regions per sample and provide an indication of the EF for each Raman mode. The $\mathrm{H}_{2}$-treated sample shows the greatest enhancement at band P1, the EF for which was evaluated to be $3.4 \times 10^{5}$. with those for neat $\mathrm{R} 6 \mathrm{G}$ and $\mathrm{W}_{18} \mathrm{O}_{49}$, which is presented in Fig. 6a. The spectrum for the hybrid features occurrence of a new band with an optical absorption onset at $\sim 580 \mathrm{~nm}$. The band locates between the band-band transition absorption edge for $\mathrm{W}_{18} \mathrm{O}_{49}, 420 \mathrm{~nm}$, and the photoabsorption threshold for R6G dye, $670 \mathrm{~nm}$, providing clear evidence for the efficient charge transfer between R6G and $\mathrm{W}_{18} \mathrm{O}_{49}$ material. This observation of high Raman enhancement on $\mathrm{W}_{18} \mathrm{O}_{49}$ may be based on the chargetransfer mechanism in an adsorbate-semiconductor system.

Moreover, oxygen vacancy may play an irreplaceable role in enriching the surface states of semiconductor to provide magnified affinity for the adsorbent-adsorbate interaction, which results in a further increase to the Raman signals for the probe molecules adsorbed on the semiconductor substrate surface through charge transfer involved in a CM mechanism. Providing with efficient charge transfer processes between the matching energy levels of adsorbed probe molecules and the semiconductor, both the polarizability tensor and the electron density distribution of the molecule would be modified, leading to the observation of non-totally symmetric SERS modes. As shown in Fig. 6b, R6G as a typical SERS probe molecule has the highestoccupied molecular orbital and lowest-unoccupied molecular orbital levels at -5.70 and $-3.40 \mathrm{eV}$, respectively ${ }^{29}$. The valence band and conduction band of semiconductor $\mathrm{W}_{18} \mathrm{O}_{49}$ locate at -7.71 and $-5.11 \mathrm{eV}$ (ref. 30),respectively, with oxygen vacancyassociated electronic state $\left(V_{\mathrm{O}}\right)$ well separated from the bottom of the $\mathrm{CB}$, located at about $0.5-1.0 \mathrm{eV}$ below the conduction band minimum $^{31}$. It can be expected that contributions from several types of thermodynamically feasible charge transfer resonance may be related to the overall Raman enhancement in our $\mathrm{W}_{18} \mathrm{O}_{49}-\mathrm{R} 6 \mathrm{G}$ system at an excitation of $532 \mathrm{~nm}$, including molecule resonance $\left(\mu_{\mathrm{mol}}\right)$ of $\mathrm{R} 6 \mathrm{G}$, exciton resonance $\left(\mu_{\mathrm{ex}}\right)$ of $\mathrm{W}_{18} \mathrm{O}_{49}$ defect states, and the photon induced charge transfer resonance $\left(\mu_{\mathrm{PICT}}\right)$ together with the ground-state charge transfer resonance $\left(\mu_{\mathrm{GSCT}}\right)$ from matched energy level between $\mathrm{W}_{18} \mathrm{O}_{49}$ and R6G molecules. These resonances will lead to a magnification of Raman scattering cross-section.

In fact, defect state $|V\rangle$ stemming from oxygen vacancies in the lattice of semiconductor has a crucial contribution to the molecular polarizability tensor via the vibronic coupling with molecular ground state $|I\rangle$ and molecular excited states $|K\rangle$, besides the conduction band state $|S\rangle$ and valence band state $\left|S^{\prime}\right\rangle$ of semiconductor (details are illustrated in Supplementary Methods). Under the incident laser frequency higher than the molecular resonance $\left(\omega_{0}>\omega_{\mathrm{IK}}\right)$, the polarizability tensor $\alpha_{\sigma \rho}$ can be expressed by a simple formula $\alpha_{\sigma \rho}=A+B+C^{32}$, where $A$ represents the contribution of the molecular resonance. $B$ and $C$ represent the contributions from the photoinduced charge
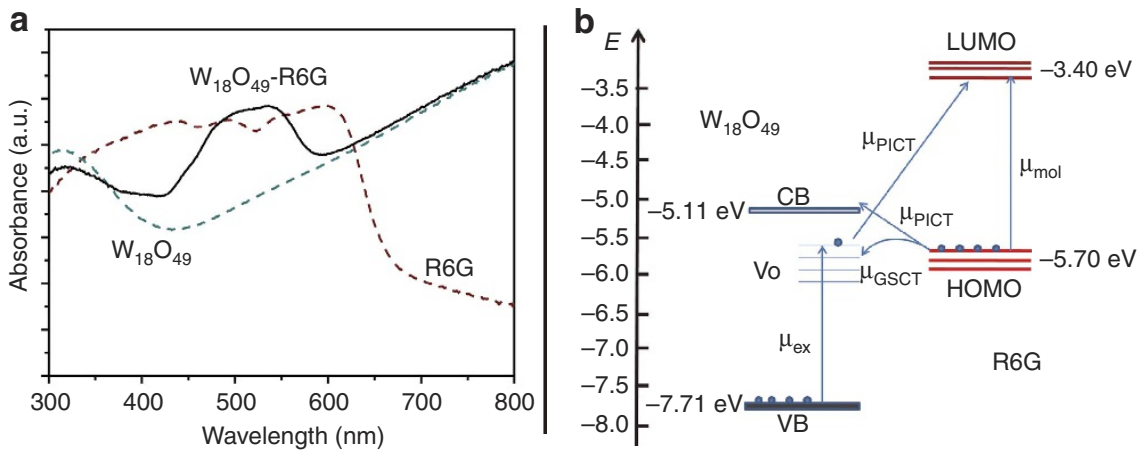

Figure 6 | Charge transfer between $\mathbf{R 6 G}$ and $\mathbf{W}_{\mathbf{1 8}} \mathbf{O}_{\mathbf{4 9}}$. (a) Absorption spectra for R6G on $\mathrm{W}_{18} \mathrm{O}_{49}$ compared with neat $\mathrm{W}_{18} \mathrm{O}_{49}$ and $\mathrm{R} 6 \mathrm{G}$ dye. (b) Energylevel diagram of $\mathrm{R} 6 \mathrm{G}$ on oxygen-deficit $\mathrm{W}_{18} \mathrm{O}_{49}$ measured in a vacuum. 
transfer of the molecule-to-semiconductor and semiconductorto-molecule, respectively, which are both related to the defect states in the semiconductor. It can be evidenced that additional possible resonant contribute to the total enhancement in defectrich semiconductor-molecule system, compared with its nondefect counterpart. When these predicted multiplicatively coincide, large EFs are expected (Supplementary Fig. 4). Thus, the observed high SERS enhancement indicates that modulation of the surface vacancy of semiconductor substrate is a simple but effective means in design of a molecule-semiconductor system with high SERS enhancement.

\section{Discussion}

In summary, metal-free SERS substrate with highly sensitive detection has been successfully fabricated employing nonstoichiometric tungsten oxide, $\mathrm{W}_{18} \mathrm{O}_{49}$ as an example, which achieved a limit detection as low as $10^{-7} \mathrm{M}$ and $\mathrm{EF}$ up to $3.4 \times 10^{5}$, an outstanding observation for semiconducting materials and even comparable to noble metal without 'hot spots'. The oxygen vacancy played a critical role in amplifying the spectroscopic signatures of probe molecules, since tungsten trioxide, $\mathrm{WO}_{3}$, only gave extremely weak signals. Moreover, the artificial creation of oxygen deficiencies by annealing the material substrate in inert or reducing atmosphere $\left(\mathrm{Ar} / \mathrm{H}_{2}\right)$ brought about further Raman enhancements, providing unambiguous evidence that the presence of oxygen vacancy, either intrinsic or postcreated ones, could help magnify the Raman signals. The extremely high SERS sensitivity on semiconducting materials can probably be attributed to the presence of oxygen deficiencies, either intrinsic or on the surface, and the resultant strengthened interaction with probe molecules via vibronic coupling. These observations provide important clues in the future strategy design of efficient semiconducting SERS substrate, and may bring important fundamental advance in material science and chemistry.

\section{Methods \\ Synthesis of sea urchin-like $\mathrm{W}_{18} \mathrm{O}_{49}$ nanowires and $\mathrm{WO}_{3}$ nanoparticles \\ on $\mathrm{Si} / \mathrm{SiO}_{2} . \mathrm{W}_{18} \mathrm{O}_{49}$ nanowires in sea urchin-like morphology on $\mathrm{Si} / \mathrm{SiO}_{2}$ substrate were prepared according to a recently published hydrothermal approach ${ }^{13}$. In brief, $\mathrm{WCl}_{6}(0.099 \mathrm{~g})$ was dissolved in $30 \mathrm{ml}$ absolute ethanol, which was transferred to a Teflon-lined stainless steel autoclave holding several horizontally oriented $\mathrm{Si} / \mathrm{SiO}_{2}$ substrates. The autoclave was then sealed and heated at $180^{\circ} \mathrm{C}$ for $12 \mathrm{~h}$. After finishing the reaction, the substrates were rinsed thoroughly with absolute ethanol and dried naturally at room temperature before use. The $\mathrm{W}_{18} \mathrm{O}_{49}$ nanowires on substrate were transformed to the corresponding trioxide form, $\mathrm{WO}_{3}$, by annealing in air. The temperature was set at $500^{\circ} \mathrm{C}$ and the sample was kept at the temperature for $1 \mathrm{~h}$.}

Modulating surface vacancy states. Modulation of the surface oxygen vacancy states was achieved through annealing the as-prepared $\mathrm{W}_{18} \mathrm{O}_{49}$ nanowires by heating in $\mathrm{Ar} / \mathrm{H}_{2}$ atmosphere at $300^{\circ} \mathrm{C}$ for $1 \mathrm{~h}$. The velocity of $\mathrm{Ar} / \mathrm{H}_{2}$ was fixed at 400 and $200 \mathrm{ml} \mathrm{min}^{-1}$, respectively.

Raman measurement. To study the Raman enhancement effect by the tungsten oxide materials, R6G dissolved in deionized water was employed to be the probe molecule because R6G has a large Raman scattering cross-section at the laser excitation wavelength applied for the SERS experiments, $532.8 \mathrm{~nm}$ (ref. 33). The tested concentration ranged from $10^{-4}$ to $10^{-7} \mathrm{M}$. Stock solution of $10^{-3} \mathrm{M}$ was initially made, and solutions of other concentrations were obtained by successive dilution by factors of $10^{1}$ or $10^{2}$. After dropping an aliquot of the respective solutions on the substrate and drying for at least $5 \mathrm{~h}$, Raman spectra were subsequently collected on a high-resolution confocal Raman spectrometer (LabRAM HR-800) using the same instrumental settings for ready comparisons. The excitation wavelength was $532.8 \mathrm{~nm}$ and a $\times 50 \mathrm{~L}$ objective was used to focus the laser beam. The spectra were acquired for $30 \mathrm{~s}$ with three accumulations and the laser power was maintained at $0.3 \mathrm{~mW}$ with an average spot size of $1 \mu \mathrm{m}$ in diameter in all acquisitions. For each sample, Raman spectra from different areas were collected, and the signal intensity was averaged for final analysis, from which relative s.d. values for EFs were estimated (Supplementary Methods and Supplementary Figs 5-8).

\section{References}

1. Chang, R. K. \& Furtak, T. E. Surface Enhanced Raman Scattering (Plenum Press, 1982).

2. Campion, A. \& Kambhampati, P. Surface-enhanced Raman scattering. Chem. Soc. Rev. 27, 241-250 (1998).

3. Nie, S. \& Emory, S. R. Probing single molecules and single nanoparticles by surface-enhanced Raman scattering. Science 275, 1102-1106 (1997).

4. Kneipp, J., Kneipp, H. \& Kneipp, K. SERS-a single-molecule and nanoscale tool for bioanalytics. Chem. Soc. Rev. 37, 1052-1060 (2008).

5. Lombardi, J. R. \& Birke, R. L. A unified view of surface-enhanced Raman scattering. Acc. Chem. Res. 42, 734-742 (2009).

6. Quagliano, L. G. Observation of molecules adsorbed on III-V semiconductor quantum dots by surface-enhanced Raman scattering. J. Am. Chem. Soc. 126, 7393-7398 (2004).

7. Li, W. et al. CuTe nanocrystals: shape and size control, plasmonic properties, and use as SERS probes and photothermal agents. J. Am. Chem. Soc. 135, 7098-7101 (2013).

8. Jiang, L. et al. Surface-enhanced Raman scattering spectra ofadsorbates on $\mathrm{Cu}_{2} \mathrm{O}$ nanospheres: charge-transfer andelectromagnetic enhancement. Nanoscale 5, 2784-2789 (2013).

9. Musumeci, A. et al. SERS of semiconducting nanoparticles $\left(\mathrm{TiO}_{2}\right.$ hybrid composites). J. Am. Chem. Soc. 131, 6040-6041 (2009).

10. Qi, D., Lu, L., Wang, L. \& Zhang, J. Improved SERS sensitivity on plasmon-free $\mathrm{TiO}_{2}$ photonic microarray by enhancing light-matter coupling. J. Am. Chem. Soc. 136, 9886-9889 (2014).

11. Li, L. et al. Metal oxide nanoparticle mediated enhanced Raman scattering and its use in direct monitoring of interfacial chemical reactions. Nano Lett. 12, 4242-4246 (2012).

12. Abe, R., Takami, H., Murakami, N. \& Ohtani, B. Pristine simple oxides as visible light driven photocatalysts: highly efficient decomposition of organic compounds over platinum-loaded tungsten oxide. J. Am. Chem. Soc. 130, 7780-7781 (2008).

13. Tian, Y. et al. Synergy of $\mathrm{W}_{18} \mathrm{O}_{49}$ and polyaniline for smart supercapacitor electrode integrated with energy level indicating functionality. Nano Lett. 14, 2150-2156 (2014).

14. Cong, S., Tian, Y., Li, Q., Zhao, Z. \& Geng, F. Single-crystalline tungsten oxide quantum dots for fast pseudocapacitor and electrochromic applications. $A d v$. Mater. 26, 4260-4267 (2014).

15. Manthiram, K. \& Alivisatos, A. P. Tunable localized surface plasmon resonances in tungsten oxide nanocrystals. J. Am. Chem. Soc. 134, 3995-3998 (2012).

16. Remškar, M. et al. $\mathrm{W}_{5} \mathrm{O}_{14}$ nanowires. Adv. Funct. Mater. 17, 1974-1978 (2007)

17. Sundberg, M. The crystal and defect structures of $\mathrm{W}_{25} \mathrm{O}_{73}$, a member of the homologous series $\mathrm{W}_{\mathrm{n}} \mathrm{O}_{3 \mathrm{n}-2}$. Acta Crystallogr. Sec. B 32, $2144-2149$ (1976).

18. Guo, C. et al. Morphology-controlled synthesis of $\mathrm{W}_{18} \mathrm{O}_{49}$ nanostructures and their near-infrared absorption properties. Inorg. Chem. 51, 4763-4771 (2012).

19. Viswanathan, K., Brandt, K. \& Salje, E. Crystal structure and charge carrier concentration of $\mathrm{W}_{18} \mathrm{O}_{49}$. J. Solid State Chem. 36, 45-51 (1981).

20. Hildebrandt, P. \& Stockburger, M. Surface-enhanced resonance Raman spectroscopy of Rhodamine 6G adsorbed on colloidal silver. J. Phys. Chem. 88, 5935-5944 (1984).

21. Le Ru, E. C., Blackie, E., Meyer, M. \& Etchegoin, P. G. Surface enhanced Raman scattering enhancement factors: a comprehensive study. J. Phys. Chem. C 111, 13794-13803 (2007).

22. Hsiao, W.-H. et al. Surface-enhanced Raman scattering imaging of a single molecule on urchin-like silver nanowires. ACS Appl. Mater. Interfaces 3, 3280-3284 (2011).

23. Lal, S., Grady, N. K., Goodrich, G. P. \& Halas, N. J. Profiling the near field of a plasmonic nanoparticle with Raman-based molecular rulers. Nano Lett. 6, 2338-2343 (2006).

24. Yang, H. et al. Comparison of surface-enhanced Raman scattering on graphene oxide, reduced graphene oxide and graphene surfaces. Carbon 62, 422-429 (2013).

25. Naldoni, A. et al. Effect of nature and location of defects on bandgap narrowing in black $\mathrm{TiO}_{2}$ nanoparticles. J. Am. Chem. Soc. 134, 7600-7603 (2012).

26. Jeon, S. \& Yong, K. Synthesis and characterization of tungsten oxide nanorods from chemical vapor deposition-grown tungsten film by low-temperature thermal annealing. J. Mater. Res. 23, 1320-1326 (2008).

27. He, X. W. et al. Memristive properties of hexagonal $\mathrm{WO}_{3}$ nanowires induced by oxygen vacancy migration. Nanoscale Res. Lett. 8, 50 (2013).

28. Xi, G. et al. Ultrathin $\mathrm{W}_{18} \mathrm{O}_{49}$ nanowires with diameters below $1 \mathrm{~nm}$ : synthesis, near-infrared absorption, photoluminescence, and photochemical reduction of carbon dioxide. Angew. Chem. Int. Ed. 51, 2395-2399 (2012).

29. Ling, X. et al. Can graphene be used as a substrate for Raman enhancement? Nano Lett. 10, 553-561 (2010).

30. Rawal, S. B. et al. Design of visible-light photocatalysts by coupling of narrow bandgap semiconductors and $\mathrm{TiO}_{2}$ : effect of their relative energy band positions on the photocatalytic efficiency. Catal. Sci. Technol. 3, 1822-1830 (2013). 
31. Wang et al. Semiconductor-to-metal transition in $\mathrm{WO}_{3-x}$ : nature of the oxygen vacancy. Phys. Rev. B 84, 073103 (2011).

32. Lombardi et al. Theory of surface-enhanced Raman scattering in semiconductors. J. Phys. Chem. C 118, 11120-11130 (2014).

33. Shim, S., Stuart, C. M. \& Mathies, R. A. Resonance raman cross-sections and vibronic analysis of Rhodamine $6 \mathrm{G}$ from broadband stimulated Raman spectroscopy. ChemPhysChem 9, 697-699 (2008).

\section{Acknowledgements}

This work was supported by the National Natural Science Foundation of China (51372266), the Natural Science Foundation of Jiangsu Province (BK20130348) and Suzhou Industrial Science and Technology Programm (ZXG201426). F.G. acknowledges support from the National Natural Science Foundation of China (51402204), Thousand Young Talents Program and Jiangsu Specially-Appointed Professor Program. Y.Z. and Q.L. acknowledge the support of the National Natural Science Foundation of China (51202282) and National Basic Research Program by Ministry of Science and Technology (2011CB932600)

\section{Author contributions}

Z.Z. and F.G. conceived the project and designed the experiments. S.C., Y.Y., Z.C., J.H., M.Y. and Y.S. performed material synthesis, structural characterization and Raman measurements. S.C., Y.Z., L.L., Q.L., F.G. and Z.Z. analysed the data. S.C., F.G. and Z.Z. co-wrote the paper. All authors discussed the results and commented on the manuscript.

\section{Additional information}

Supplementary Information accompanies this paper at http://www.nature.com/ naturecommunications

Competing financial interests: The authors declare no competing financial interests.

Reprints and permission information is available online at http://npg.nature.com/ reprintsandpermissions/

How to cite this article: Cong, S. et al. Noble metal-comparable SERS enhancement from semiconducting metal oxides by making oxygen vacancies. Nat. Commun. 6:7800 doi: 10.1038/ncomms8800 (2015).

(c) (i) This work is licensed under a Creative Commons Attribution 4.0 International License. The images or other third party material in this article are included in the article's Creative Commons license, unless indicated otherwise in the credit line; if the material is not included under the Creative Commons license, users will need to obtain permission from the license holder to reproduce the material. To view a copy of this license, visit http://creativecommons.org/licenses/by/4.0/ 